\title{
DETECTION OF POWER-LINES IN COMPLEX NATURAL SURROUNDINGS
}

\author{
Rajeev M Bhujade ${ }^{1}$, Adithya $\mathrm{V}^{2}$, Hrishikesh $\mathrm{S}^{3}$, Balamurali $\mathrm{P}^{4}$ \\ ${ }^{1,2,3,4}$ Bangalore Innovation Lab, Tata Consultancy Services, Bangalore, India. \\ rajeev.b@tcs.com, adithya.v@tcs.com, hrishikesh.sharma@tcs.com, \\ balamurali.petcs.com
}

\begin{abstract}
Power transmission line inspection using Unmanned Aerial Vehicles (UAV) is taking off as an exciting solution due to advances in sensors and flight technology. Extracting power-lines from aerial images, taken from the UAV, having complex natural surroundings is a critical task in the above problem. In this paper we propose an approach for suppressing natural surrounding that leads to power line detection. The results of applying our method on real-life video frames taken from a UAV demonstrate that our approach is very effective. We believe that our approach can be easily used for line detection in any other real outdoor video as well.
\end{abstract}

\section{KEYWORDS}

Transmission line extraction, Background Suppression, UAV

\section{INTRODUCTION}

Automatic monitoring of transmission lines using unmanned aerial vehicles (UAV) is an upcoming application. Power-lines are exposed to natural environmental factors like strong winds, ageing, interference of neighbouring vegetation e.g. trees etc. Hence the power distribution grid requires regular monitoring for uninterrupted power supply. Among the methods for powerline monitoring, manual inspection from the ground, using helicopters, crawling robots and satellites were considered. Manual inspection is slow and cannot be done over all terrains. Helicopters flying close to power-lines present a definite hazard to the crew and are costly too. Crawling robots too are slow and cannot be used when power is switched off. Satellites cannot have an on-demand motion schedule. With the improvements in flight algorithms and development of light-weight cameras, it is now possible to have a small UAV carrying a lightweight camera to capture representative images or a video of the power-lines as it flies closely along the length of the grid.

The image capturing can be done using various cameras e.g. visual or near infrared camera. The popular imaging is done by using visual camera. Detection of transmission lines in visual-band video taken by UAV is an important step for real time UAV navigation and automatic line fault detection. The power-lines run long distances over different terrains. The image background 
hence can vary from trees and patches of greenery to different flat spaces along with other objects like houses and roads. Detection of power-lines over these terrains is challenging yet their monitoring is mandatory by various government laws. Also in places where GPS signal may not be available for navigation, navigation along detected lines could be almost mandatory.

In past, some work has been done on power-line detection over complex natural surroundings. Li et al. [1] used a modified pulse coupled neural network to suppress the complex background. Edge detection based approach using Marr-Hildreth detection algorithm is used in [2] by Tonget al. A knowledge based approach has been proposed in [3] by Li et al. that uses clustering after Hough transform to detect the power-lines. Yuee et al. in [4] uses a steerable filter for realtime detection of power-lines. However, these papers operate on data gathered by downward looking cameras.

In this paper, we propose an approach to power-line detection from color video frames obtained by a front looking camera. The proposed method is tested on real video data captured from a UAV platform in rural areas near Bangalore, and experimental results are discussed.

\section{PROPOSED APPROACH}

We have designed a detection algorithm consists of the following four stages. The first stage is aimed at removing sky from the background. Since at the core, we use the intensity of reflected light from the power line for its detection, we need to first remove the large regions of sky of similar or higher brightness, which we found to be interfering with power line detection. The second stage consists of removal of vegetation from the remaining background. This is needed since if present, it contributes far too many unnecessary dense clusters of edges during the core, final stage of line detection. After removal of sky and vegetation, certain habitats or other structures can still remain in the background, within the field of view of the camera. The third stage uses a morphological erosion operator that suppresses small and large patches in the thresholded binary image. The fourth stage consists of using Hough transform that connects power line pixels and detects the power line. Currently, we have worked out first two stages fully. In this paper, we focus on mainly describing the first two stages which have novel approach. We do touch upon initial results of the remaining two stages.

\subsection{Colour based Suppression}

The frames captured by the camera are in RGB format. The RGB color space is correlated.Hence a need arises for a better and sparse representation of the image data. Sparseness is also required since any real-time transmission of images/video to the ground control station (GCS) over bandwidth-constrained link or network necessitates compression of data. We shortlisted two recent and popular sparse representation spaces in image processing: Hue-Saturation-Value (HSV) color space and Hue-Saturation-Lightness (HSL) for consideration. We found that HSV is more intuitive for the purpose of background suppression. For recapitulation, Hue is the dominant color component at the location of interest while the Saturation is the color intensity (or purity) and Value translates to the luminance or simple brightness.

From the experimental data, we found that the dominant background of the images in the powerline images are the sky and the greenery, as expected. It is obvious that both these components have specific HSV values that do not interfere with the power-line values. The other types of 
background like roads and houses can have overlapping HSV values with powerlines and hence cannot be suppressed by colour based suppression.

\subsection{Suppression of Sky}

During daytime, sky is light blue to white in color. This is because of Rayleigh scattering effect [5]. In evenings, it can have reddish hue, again due to Rayleigh scattering. It has good brightness levels i.e. the value. For sky suppression we can supress all values in the image having saturation greater than $39 \%$ irrespective of hue and value. This threshold is tuneable to other illumination levels as per the data, but the fact that saturation levels of line and sky remain discernible holds still.

\subsection{Suppression of Vegetation}

The next important step in our algorithm is that of vegetation removal. Such greenery includes vegetation like grass, scrubs, tree canopy etc. The low saturation levels in the green hue range tend to suppress the power-lines as the thin lines appear to reflect some of the background colour and hence are to be left untouched. Once again, for greenery suppression, we supress all values in the image having saturation greater than same threshold i.e. $39 \%$.

After sky and greenery suppression, the HSV image is first converted to a grey scale image and then a binary image using a suitable threshold.

\subsection{Erosion of Remaining Background}

The colour based suppression reduces greenery and sky, but some interference from roads and houses can be present at times. These appear as patches in the foreground of the binary image, as compared to the straight and thin power-lines. We use erosion operator from morphology [6] to remove such vestigial background. The kernel of the background is an all-1 matrix. The thresholding is specific to power line detection. When the kernel is moved onto a region centered on a pixel, and the corresponding condition is satisfied, then the center pixel is retained as foreground pixel, else it is eroded.

This morphological operation leaves the thin power-lines unchanged while eroding large patches from the inside. The eroded output is then fed to the next stage of the Line Hough transform.

\subsection{Hough Transform}

Hough transform is a very popular technique used for identifying lines in binary images. For a pixel $(\mathrm{x}, \mathrm{y})$ in the image, the equation of a line passing through it is given as [7],

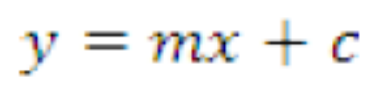

where $\mathrm{m}$ is the slope of the line and $\mathrm{c}$ is the $\mathrm{y}$-intercept. Alternately, in polar coordinate, the line can be represented is as, 


$$
\rho=x \cos \theta+y \sin \theta
$$

where $\rho$ is the length of the perpendicular from the origin to the line and $\theta$ is the angle between the perpendicular and $\mathrm{x}$-axis. Thus for a given pixel, in the polar coordinates, a line is represented by a point and by varying the value of $\theta$ from 0 to 360 we get a sinusoidal wave. Doing this for other pixels, we get different sinusoidal waves which intersect at points representing pixels that lie on a single line. Brighter the point of intersection, stronger is the line in the image.

The number of intersections is calculated by a voting system in a $\rho$ by $\theta$ accumulator. For every line passing through a pixel, a vote is cast in the corresponding $(\rho, \theta)$ bin. A threshold is set for number of votes that are required for accepting a line. The classical Hough transform operates on all the bright pixels in the binary image. This is a tedious process, operating one by one on every bright pixel. The output is a set of $(\rho, \theta)$ pairs. We use a particular variation of Hough transform, progressive probabilistic Hough transform given by Matas et al. [8], that performs faster.

The Hough threshold and minimum line length are fixed based on the expected length of the power-line in the image. The gap threshold depends on the average power-line pixel intensity.

\section{EXPERIMENT AND DISCUSSION}

To test our method we use a short RGB video file of 880 frames and a resolution of $640 \times 480$ captured by a front facing camera from a UAV flying above the power-lines in rural Bangalore. The background includes greenery, sky, roads and houses. The algorithm is written in $\mathrm{C}++$ using OpenCV libraries. OpenCV library is a cross-platform library free for use under OpenBSD

license and supporting real-time image processing. We have used the $\mathrm{C}++$ library [9]. The algorithm is as follows:

1) The RGB image is converted to HSV image

2) The sky and greenery suppression are done making the pixels having HSV values in the corresponding ranges black.

3) The HSV is converted to grey scale image and a simple threshold of 128 is used to convert the grey scale image to a binary image. The threshold is chosen as half the maximum pixel intensity to pick up maximum line pixels as power-lines appear bright due to reflection to the UAV from above.

4) Morphology erosion is done next.

5) The probability Hough transform function in OpenCV based on the technique by Matas et al. [5] is used. The different threshold values used are, minimum-line-length $=70$ pixels, Hough threshold $=50$ pixels and gap threshold $=5$ pixels.

The parameters that we tuned for this detection are tabulated as follows. 
Table 1. Parameter Tuning

\begin{tabular}{|l|c|c|}
\hline \multicolumn{1}{|c|}{ Name } & Tuning Range & Tuned Value \\
\hline Saturation threshold & $0-50 \%$ & $39 \%$ \\
\hline Hough: min. line length & $40-90$ & 70 \\
\hline Hough: threshold & $60-120$ & 100 \\
\hline Hough: max. gap threshold & $2-15$ & 5 \\
\hline
\end{tabular}

To illustrate the image processing, consider figure 1(a), which is a frame in the captured outdoor video. It has four power-lines and the background comprises of a white sky, greenery, roads and some houses. It was observed that the saturation range for bright objects in the image, like sky and power-lines lie between 0 to $50 \%$ beyond which the concentration of the colors become more significant. A value of $39 \%$ was chosen for maximum sky elimination while retaining the powerlines. This value was able to eliminate most of the greenery also, as shown in figure 1(b).

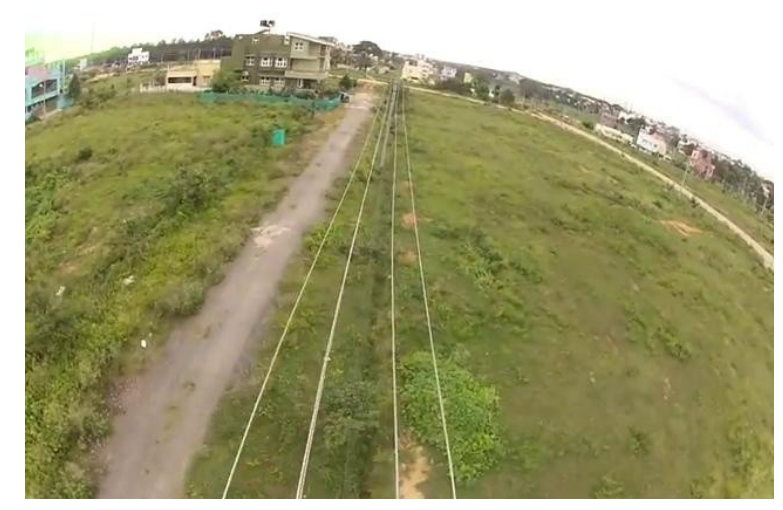

Figure. 1(a): A Frame in the captured video

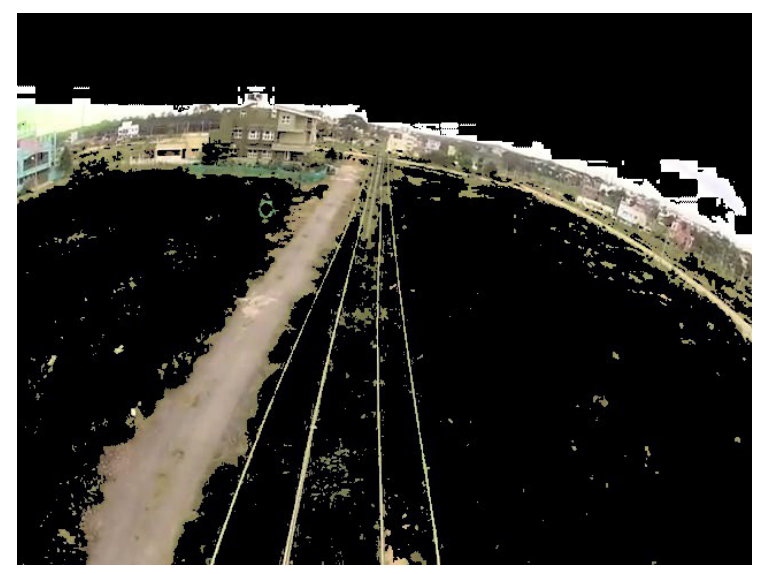

Figure. 1(b): Sky and greenery suppression.

The figures 1(c) and 1(d) show the outputs after binary thresholding and erosion operation. The erosion removes the road and other background structures to a large extent while retaining the 
power lines. It can be observed clearly that background suppression technique used is able to significantly reduce background clutter.

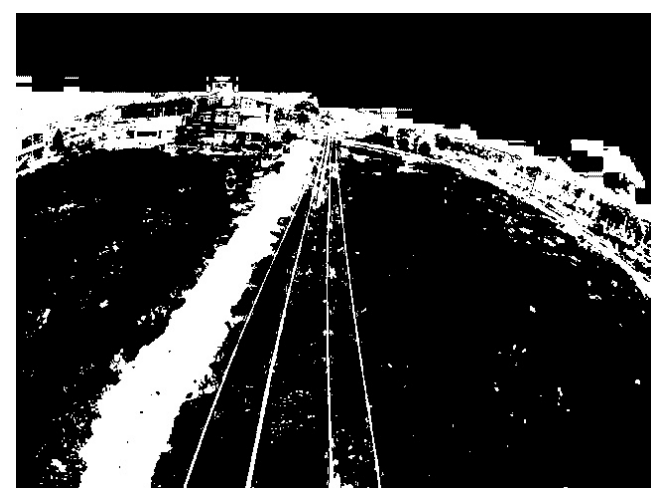

Figure. 1(c): Thresholded image.

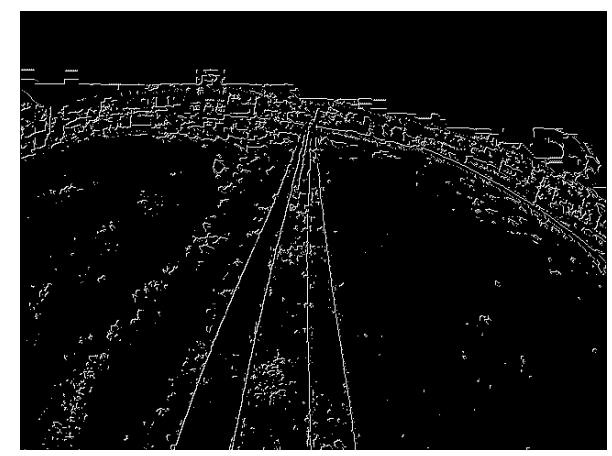

Figure. 1(d): Output after Erosion operation.

The prominence of the power-lines in the image compared to the discontinuous patches of the background still retained after the erosion operation allows us to choose a higher threshold for minimum Hough line length and Hough threshold and a lower value for the gap threshold which is essentially the maximum pixel gap to be ignored while connecting the bright points. The output of Hough transform stage for detection of linear shapes is shown in Figure 1(e).

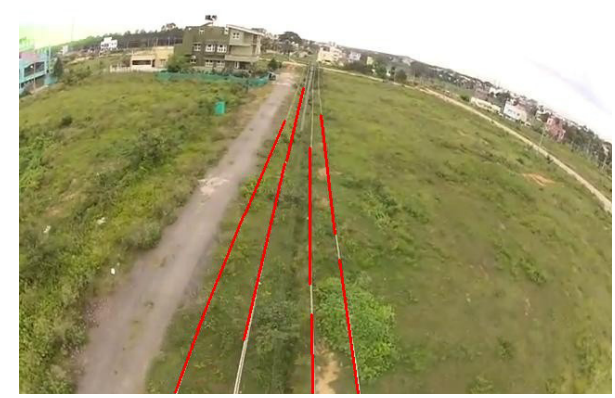

Figure. 1(e): Detected power-lines 
In the figure, the red lines show the detected power-line segments. The discontinuities in the lines occur due to the slight differences in the line orientation and displacement. This is caused by non-uniform line thickness across the image in this view which depends on the UAV and camera position and orientation at that instant and also due to some residual background clutter.

\section{CONCLUSION}

In this paper, we have presented a new method for complex background suppression on video frames captured by a front facing camera mounted on a UAV. The combination of sky and greenery removal, followed by morphological erosion, contributes significantly in elimination of majority of the background. The application of Hough transform to this image demonstrates that power-line detection is possible. Future work will be focussed on using information across frames for even better/more robust detection of the power-lines. We are hopeful that our methods of complex background removal, which show good results, will find utility in many other image/video processing applications.

\section{ACKNOWLEDGEMENTS}

We thank Prof. Omkar and his team from Indian Institute of Science, Bangalore, for providing us with test video data to run our algorithm.

\section{REFERENCES}

[1] Zhengrong Li, Yuee Liu, Rodney Walker, Ross Hayward, "Towards Automatic Power Line Detection for UAV Surveillance System Using Pulse Coupled Neural Filter and Hough Transform" Machine Vision and Applications, 2009.

[2] Wei-Guo Tong, Bao-Shuli-, Jin-shayuan, Shu-Tao Zhao, "Transmission Line Extraction and Recognition from Natural Complex Background," Proceedings of the Eighth International Conference on Machine Learning and Cybernetics, Baoding, 12-15 July 2009.

[3] Zhengrong Li, Hayward, R.,Jinglan Zhang, Jinhai Cai, "Knowledge-based power line detection for UAV surveillance and inspection systems," 23rd International Conference on Image and Vision Computing, 2008.

[4] Lie Yuee, Mejias Luis\& Li Zhenrong, "Fast Power Line Detection and Localization using Steerable Filter for active UAV Guidance," 12th International Society for Photogrammetry \& Remote Sensing, (ISPRS2012).

[5] Pedro Lilienfeld, "A Blue Sky History." (2004). Optics and Photonics News. Vol. 15, Issue 6, pp. $32-39$.

[6] "A Novel Erosion Operator", TCS Internal, 2013.

[7] "Image Transforms - Hough Transform," Homepages.inf.ed.ac.uk. Retrieved 2009-08-17.

[8] J. Matas, C. Galambos, J Kittler, "Robust Detection of Lines Using the Progressive Probabilistic Hough Transform," Computer Vision and Image Understanding 78, 119-137 (2000).

[9] OpenCV reference manual: http://docs.opencv.org 


\section{AUTHORS}

Rajeev M. Bhujade obtained his master's degree from Indian Institute of Technology, Bombay, in 2002.Since then, he has been part of R\&D team at Innovation Labs at TCS Bangalore. His research interests include audio signal processing and communication, and recently, computer vision.

Adithya V. obtained her master's degree from Indian Institute of Technology, Bombay, in 2012. She currently works for Innovation Labs, Bangalore as a researcher. Her research interests include image processing algorithms.

Hrishikesh Sharma obtained his bachelor's and master's degree from Indian Institute of Technology, Bombay, in 1999, and a doctorate from the same place in 2012. He works as a scientist with Innovation Labs, Tata Consultancy Services Ltd., Bangalore. His current research interests include image processing and computer vision systems and algorithms.

Balamurali P. obtained his doctorate from Aalborg university in 2013. He is the research head of Innovation Labs, TCS Bangalore. His research interests include multiple areas of digital communication and VLSI, especially communication networks. 